Network Working Group

Request for Comments: 1361
D. Mills

University of Delaware

August 1992

\title{
Simple Network Time Protocol (SNTP)
}

Status of this Memo

This memo provides information for the Internet community. It does not specify an Internet standard. Distribution of this memo is unlimited.

Abstract

This memorandum describes the Simple Network Time Protocol (SNTP), which is an adaptation of the Network Time Protocol (NTP) used to synchronize computer clocks in the Internet. SNTP can be used when the ultimate performance of the full NTP implementation described in RFC-1305 is not needed or justified. It involves no change to the current or previous NTP specification versions or known implementations, but rather a clarification of certain design features of NTP which allow operation in a simple, stateless RPC mode with accuracy and reliability expectations similar to the UDP/TIME protocol described in $\mathrm{RFC}-868$.

This memorandum does not obsolete or update any RFC. A working knowledge of RFC-1305 is not required for an implementation of SNTP.

1. Introduction

The Network Time Protocol (NTP) specified in RFC-1305 [MIL92] is used to synchronize computer clocks in the global Internet. It provides comprehensive mechanisms to access national time and frequency dissemination services, organize the time-synchronization subnet and adjust the local clock in each participating subnet peer. In most places of the Internet of today, NTP provides accuracies of 1-50 ms, depending on the jitter characteristics of the synchronization source and network paths.

RFC-1305 specifies the NTP protocol machine in terms of events, states, transition functions and actions and, in addition, optional algorithms to improve the timekeeping quality and mitigate among several, possibly faulty, synchronization sources. To achieve accuracies in the low milliseconds over paths spanning major portions of the Internet of today, these intricate algorithms, or their functional equivalents, are necessary. However, in many cases accuracies of this order are not required and something less, perhaps 
in the order of one second, is sufficient. In such cases simpler protocols such as the Time Protocol [POS83], have been used for this purpose. These protocols usually involve a remote-procedure call (RPC) exchange where the client requests the time of day and the server returns it in seconds past some known reference epoch.

NTP is designed for use by clients and servers with a wide range of capabilities and over a wide range of network delays and jitter characteristics. Most members of the Internet NTP synchronization subnet of today use software packages including the full suite of NTP options and algorithms, which are relatively complex, real-time applications. While the software has been ported to a wide variety of hardware platforms ranging from supercomputers to personal computers, its sheer size and complexity is not appropriate for many applications. Accordingly, it is useful to explore alternative access strategies using far simpler software appropriate for accuracy expectations in the order of a second.

This memorandum describes the Simple Network Time Protocol (SNTP), which is a simplified access strategy for servers and clients using NTP as now specified and deployed in the Internet. There are no changes to the protocol or implementations now running or likely to be implemented in the near future. The access paradigm is identical to the UDP/Time Protocol and, in fact, it should be easily possible to adapt a UDP/Time client implementation, say for a personal computer, to operate using SNTP. Moreover, SNTP is also designed to operate in a dedicated server configuration including an integrated radio clock. With careful design and control of the various latencies in the system, which is practical in a dedicated design, it is possible to deliver time accurate to the order of microseconds.

It is strongly recommended that SNTP be used only at the extremities of the synchronization subnet. SNTP clients should operate only at the leaves (highest stratum) of the subnet and in configurations where no SNTP client is dependent on another SNTP client for synchronization. SNTP servers should operate only at the root (stratum 1) of the subnet and then only in configurations where no other source of synchronization other than a reliable radio clock is available. The full degree of reliability ordinarily expected of primary servers is possible only using the redundant sources, diverse subnet paths and crafted algorithms of a full NTP implementation. This extends to the primary source of synchronization itself in the form of multiple radio clocks and backup paths to other primary servers should the radio clock fail or become faulty. Therefore, the use of SNTP rather than NTP in primary servers should be carefully considered. 


\section{NTP Timestamp Format}

SNTP uses the standard NTP timestamp format described in RFC-1305 and previous versions of that document. In conformance with standard Internet practice, NTP data are specified as integer or fixed-point quantities, with bits numbered in big-endian fashion from zero starting at the left, or high-order, position. Unless specified otherwise, all quantities are unsigned and may occupy the full field width with an implied zero preceding bit zero.

Since NTP timestamps are cherished data and, in fact, represent the main product of the protocol, a special timestamp format has been established. NTP timestamps are represented as a 64-bit unsigned fixed-point number, in seconds relative to oh on 1 January 1900. The integer part is in the first 32 bits and the fraction part in the last 32 bits. This format allows convenient multiple-precision arithmetic and conversion to Time Protocol representation (seconds), but does complicate the conversion to ICMP Timestamp message representation (milliseconds). The precision of this representation is about 200 picoseconds, which should be adequate for even the most exotic requirements.

Note that since some time in 1968 the most significant bit (bit 0 of the integer part) has been set and that the 64-bit field will overflow some time in 2036. Should NTP or SNTP be in use in 2036, some external means will be necessary to qualify time relative to 1900 and time relative to 2036 (and other multiples of 136 years). Timestamped data requiring such qualification will be so precious that appropriate means should be readily available. There will exist a 200-picosecond interval, henceforth ignored, every 136 years when the 64-bit field will be zero, which by convention is interpreted as an invalid timestamp.

3. NTP Message Format

Both NTP and SNTP are clients of the User Datagram Protocol (UDP) [POS83], which itself is a client of the Internet Protocol (IP) [DAR81]. The structure of the IP and UDP headers is described in the relevant specification documents and will not be described further in this memorandum. Following is a description of the SNTP message format, which follows the IP and UDP headers. The SNTP message format is identical to the NTP format described in RFC-1305, with the exception that some of the data fields are "canned," that is, initialized to prespecified values. The format of the NTP message data area, which immediately follows the UDP header, is shown below. 


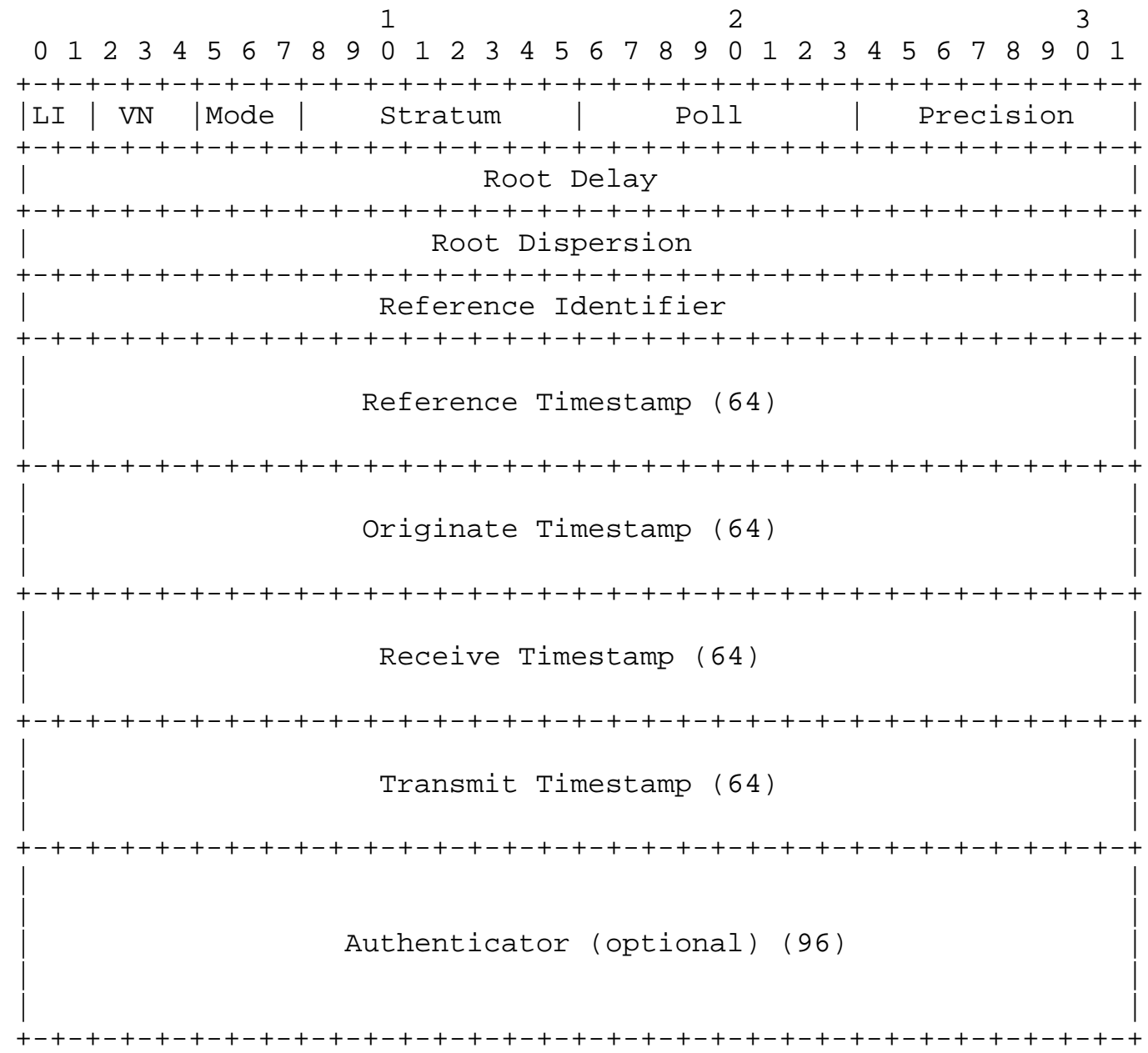

As described in the next section, in SNTP most of these fields are initialized with prespecified data. For completeness, the function of each field is briefly summarized below.

Leap Indicator (LI): This is a two-bit code warning of an impending leap second to be inserted/deleted in the last minute of the current day, with bit 0 and bit 1 , respectively, coded as follows:

$\begin{array}{lll}\text { LI } & \text { Value } & \text { Meaning } \\ - & & \\ 00 & 0 & \text { no warning } \\ 01 & 1 & \text { last minute has } 61 \text { seconds } \\ 10 & 2 & \text { last minute has } 59 \text { seconds) } \\ 11 & 3 & \text { alarm condition (clock not synchronized) }\end{array}$




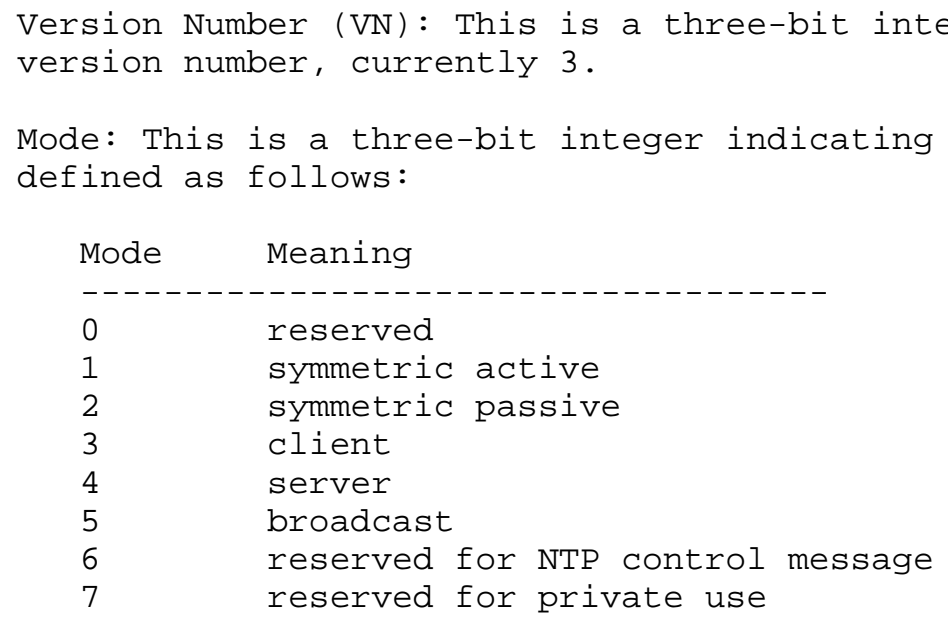

The use of this field will be discussed in the next section.

Stratum: This is a eight-bit integer indicating the stratum level of the local clock, with values defined as follows:

$\begin{array}{ll}\text { Stratum } & \text { Meaning } \\ ------ & \text { unspecified or unavailable } \\ 0 & \text { primary reference (e.g., radio clock) } \\ 1 & \text { secondary reference (via NTP or SNTP) } \\ 2-15 & \text { reserved }\end{array}$

Poll Interval: This is an eight-bit signed integer indicating the maximum interval between successive messages, in seconds to the nearest power of two. The values that normally appear in this field range from 6 to 10 , inclusive.

Precision: This is an eight-bit signed integer indicating the precision of the local clock, in seconds to the nearest power of two. The values that normally appear in this field range from -6 for mains-frequency clocks to -18 for microsecond clocks found in some workstations.

Root Delay: This is a 32-bit signed fixed-point number indicating the total roundtrip delay to the primary reference source, in seconds with fraction point between bits 15 and 16 . Note that this variable can take on both positive and negative values, depending on the relative time and frequency errors. The values that normally appear in this field range from negative values of a few milliseconds to positive values of several hundred milliseconds. 
Root Dispersion: This is a 32-bit unsigned fixed-point number indicating the maximum error relative to the primary reference source, in seconds with fraction point between bits 15 and 16 . The values that normally appear in this field range from zero to several hundred milliseconds.

Reference Clock Identifier: This is a 32-bit code identifying the particular reference clock. In the case of stratum 0 (unspecified) or stratum 1 (primary reference), this is a four-octet, left-justified, zero-padded ASCII string. While not enumerated as part of the NTP specification, the following are representative ASCII identifiers:

\section{Stratum Code Meaning}

0 ascii

aciis
(Automated Computer Time Service), TIME (UDP/Time Protocol), TSP (TSP Unix time protocol), DTSS

$\begin{array}{ll}1 & \text { ATOM } \\ 1 & \text { VLF } \\ 1 & \text { Callsign } \\ 1 & \text { LORC } \\ 1 & \text { GOES } \\ 1 & \text { GPS } \\ 2 & \text { address }\end{array}$
(Digital Time Synchronization Service), etc. calibrated atomic clock VLF radio (OMEGA, etc.)

Generic radio LORAN-C radionavigation system Geostationary Operational Environmental Satellite Global Positioning Service secondary reference (four-octet Internet address of the NTP server)

Reference Timestamp: This is the local time at which the local clock was last set or corrected, in 64-bit timestamp format.

Originate Timestamp: This is the local time at which the request departed the client for the server, in 64-bit timestamp format.

Receive Timestamp: This is the local time at which the request arrived at the server, in 64-bit timestamp format.

Transmit Timestamp: This is the local time at which the reply departed the server for the client, in 64-bit timestamp format.

Authenticator (optional): When the NTP authentication mechanism is implemented, this contains the authenticator information defined in Appendix C of RFC-1305. In SNTP this field is ignored for incoming messages and is not generated for outgoing messages. 


\section{SNTP Client Operations}

The model for an SNTP client operating with either an NTP or SNTP server is a RPC client with no persistent state. The client initializes the SNTP message header, sends the message to the server and strips the time of day from the reply. For this purpose all of the message-header fields shown above are set to zero, except the first octet. In this octet the Leap Indicator is set to zero (no warning) and the Mode to 3 (client). The Version Number must agree with the software version of the NTP or SNTP server; however, NTP Version 3 (RFC-1305) servers will also accept Version 2 (RFC-1119) and Version 1 (RFC-1059) messages, while NTP Version 2 servers will also accept NTP Version 1 messages. Version 0 (original NTP described in RFC-959) messages are no longer supported. Since there are NTP servers of all three versions operating in the Internet of today, it is recommended that the Version Number field be set to one.

The server reply includes all the fields described above; however, in SNTP only the Transmit Timestamp has explicit meaning. The integer part of this field contains the server time of day in the same format as the Time Protocol. While the fraction part of this field will usually be valid, the accuracy achieved with the SNTP mode of access probably does not justify its use.

The following table is a summary of the SNTP client operations. There are three recommended error checks shown in the table. In all NTP versions, if the Leap Indicator field is 3 or the Transmit Timestamp is zero (unsynchronized), the server has never synchronized or not synchronized to a valid timing source within the last 24 hours. If the Stratum field is 0 (unspecified or unavailable), the server has never synchronized, has lost reachability with all timing sources or is synchronized by some protocol other than NTP. Whether to believe the transmit timestamp or not in this case is at the discretion of the client implementation. 


\begin{tabular}{|c|c|c|}
\hline Field Name & Request & Reply \\
\hline Leap Indicator (LI) & 0 & $\begin{array}{l}\text { if } 3 \text { (unsynchronized), } \\
\text { disregard }\end{array}$ \\
\hline Version Number (VN) & (see text) & ignore \\
\hline Mode & 3 (client) & ignore \\
\hline Stratum & 0 & $\begin{array}{l}\text { if } 0 \text { (unspecified), } \\
\text { disregard }\end{array}$ \\
\hline Poll & 0 & ignore \\
\hline Precision & 0 & ignore \\
\hline Root Delay & 0 & ignore \\
\hline Root Dispersion & 0 & ignore \\
\hline Reference Identifier & 0 & ignore \\
\hline Reference Timestamp & 0 & ignore \\
\hline Originate Timestamp & 0 & ignore \\
\hline Receive Timestamp & 0 & ignore \\
\hline Transmit Timestamp & 0 & $\begin{array}{l}\text { time of day (seconds only); } \\
\text { if } 0 \text { (unsynchronized), } \\
\text { disregard }\end{array}$ \\
\hline Authenticator & (not used) & ignore \\
\hline
\end{tabular}

5. SNTP Server Operations

The model for an SNTP server operating with either an NTP or SNTP client is an RPC server with no persistent state. The SNTP server ignores all header fields except the first octet, modifies certain fields and returns the message to the sender. Since an SNTP server ordinarily does not implement the full set of NTP algorithms intended to support the highest quality service, it is recommended that an SNTP server be operated only in conjunction with a source of outside synchronization, such as a radio clock. In this case the server always operates at stratum 1 .

The first octet is interpreted as follows. The Leap Indicator and Version Number fields are ignored. Optionally, messages with version numbers other than 1, 2, or 3 can be discarded. For primary servers connected to a functioning radio clock, the Leap Indicator field is set to zero and the Stratum field is set to one in the reply. otherwise, these fields are set to 3 and zero, respectively. In any case the Version Number and Poll fields are copied intact to the reply message header. If The Mode field is set to 3 (client), it is changed to 4 (server) in the reply; otherwise, this field is set to 2 (symmetric passive).

The Stratum field is set to reflect the maximum reading error of the local clock. For all practical cases it is computed as the negative of the number of significant bits to the right of the decimal point in the NTP timestamp format. The Root Delay and Root Dispersion 
fields are set to zero for a primary server; optionally, the Root Dispersion can be set to a value corresponding to the expected (constant) maximum expected error of the primary reference source. The Reference Identifier is set to designate the primary reference source, as indicated in the table above. If this information is unspecified or unavailable, the field is set to zero.

The timestamp fields are set as follows. The Reference Timestamp, Receive Timestamp and Transmit Timestamp fields are set to the time of day at the server. The Originate Timestamp field is copied unchanged from the request. The following table summarizes these actions.

$\begin{array}{lll}\text { Field Name } & \text { Request } & \text { Reply } \\ - & \text { ignore } & \text { (normal), } 3 \\ \text { Leap Indicator (LI) } & \text { (unsynchronized) } \\ \text { Version Number (VN) } & \text { ignore } & \text { copied from request } \\ \text { Mode } & \text { (see text) } & \text { (see text) } \\ \text { Stratum } & \text { ignore } & \text { server stratum (1) } \\ \text { Poll } & \text { ignore } & \text { copied from request } \\ \text { Precision } & \text { ignore } & \text { server precision } \\ \text { Root Delay } & \text { ignore } & \text { 0 } \\ \text { Root Dispersion } & \text { ignore } & \text { (see text) } \\ \text { Reference Identifier } & \text { ignore } & \text { source identifier or } 0 \\ \text { Reference Timestamp } & \text { ignore } & \text { time of day or 0 } \\ \text { Originate Timestamp } & \text { ignore } & \text { copied from request } \\ \text { Receive Timestamp } & \text { ignore } & \text { time of day or 0 } \\ \text { Transmit Timestamp } & \text { ignore } & \text { time of day or } 0 \\ \text { Authenticator } & \text { ignore } & \text { (not used) }\end{array}$

6. References

[DAR81] Postel, J., "Internet Protocol - DARPA Internet Program Protocol specification", RFC 791, DARPA, September 1981.

[MIL92] Mills, D., "Network Time Protocol (Version 3) Specification, Implementation and Analysis", RFC 1305, University of Delaware, March 1992 .

[POS80] Postel, J., "User Datagram Protocol", RFC 768, USC/Information Sciences Institute, August 1980.

[Pos83] Postel, J., and K. Harrenstien, "Time Protocol", RFC 868, USC/Information Sciences Institute, SRI, May 1983. 


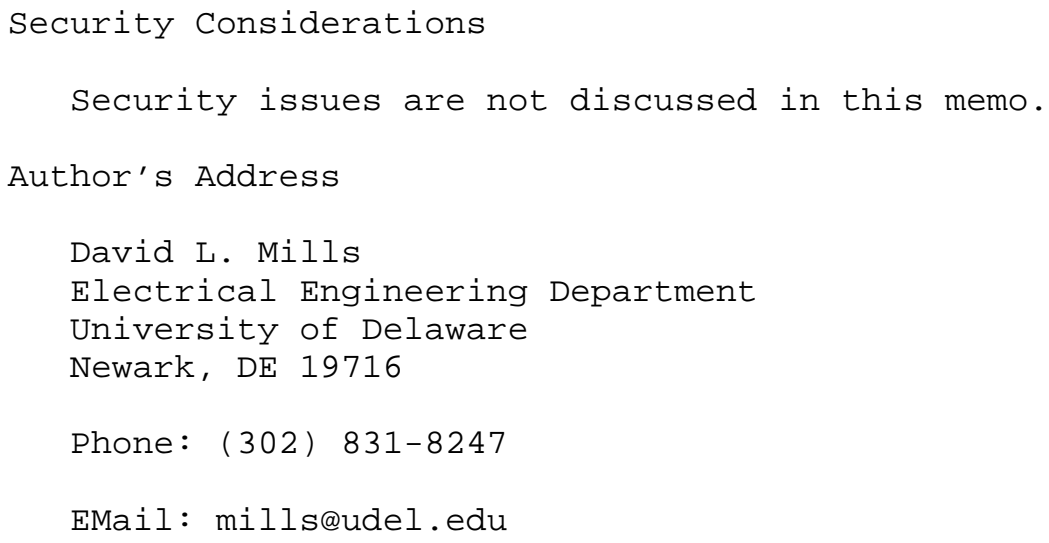

